\title{
Neural Networks Simulation of the Transport of Contaminants in Groundwater
}

\author{
Enrico Zio \\ Energy Department, Nuclear Engineering Section, Polytechnic of Milan, \\ Via Ponzio 34/3 20133 Milan, Italy \\ E-mail :enrico.zio@polimi.it \\ www.energia.polimi.it
}

Received: 01/04/09

Accepted: 07/07/09

\begin{abstract}
The performance assessment of an engineered solution for the disposal of radioactive wastes is based on mathematical models of the disposal system response to predefined accidental scenarios, within a probabilistic approach to account for the involved uncertainties. As the most significant potential pathway for the return of radionuclides to the biosphere is groundwater flow, intensive computational efforts are devoted to simulating the behaviour of the groundwater system surrounding the waste deposit, for different values of its hydrogeological parameters and for different evolution scenarios. In this paper, multilayered neural networks are trained to simulate the transport of contaminants in monodimensional and bidimensional aquifers. The results obtained in two case studies indicate that the approximation errors are within the uncertainties which characterize the input data.
\end{abstract}

Keywords: Neural networks, performance assessment of radioactive waste repositories, groundwater contaminant transport, advection-dispersion.

\section{Introduction}

The demonstration of the safe disposal of radioactive wastes over the required long time horizons is regarded as a prerequisite for the further development of nuclear energy programmes in many Countries. ${ }^{1}$ The presence of long-lived fission products and actinides in the waste imposes confinement times of the order of many thousands of years. ${ }^{2}$

The sites suitable for hosting repositories for the confinement of nuclear wastes must be characterized by hydro-geologic properties which guarantee their safe performance. ${ }^{3}$ The danger is that the contamination carried by the percolating water through the engineered containment barriers reaches the groundwater, which represents the most important vector for the transport of contaminants to the biosphere. ${ }^{1}$
The uncertainties in the hydro-geological characterization of the disposal sites and in its future evolution are such that the analysis of the safety performance of waste repositories requires an enveloping probabilistic approach based on predictive mathematical models for representing the system behaviour for many values of the uncertain hydrogeological parameters and in many different evolution scenarios, normal as well as altered by stochastic phenomena like earthquakes, tectonic displacements, human intrusions and the like, which can accelerate migration of the radionuclides towards the biosphere. ${ }^{4-5}$

Among the various models of groundwater contaminant transport, in this paper we focus on the well known advection-dispersion model, based upon the energy and mass conservation laws and the assumption of validity of Fick's law to describe the mechanisms of hydrodynamic dispersion. ${ }^{6-7}$ 
The numerous model evaluations required by the probabilistic performance assessment typically lead to very long computing times. ${ }^{8}$ For this reason, it is desirable to find some efficient algorithms to obtain reasonably approximate outputs in a short time, with the approximation errors being bounded by the uncertainty resulting in the output from the propagation of the input uncertainty.

In this paper, neural networks ( $\mathrm{NN})$ are developed for the fast, approximate solution of the advectiondispersion model. The networks are trained to predict the space-time contaminant distribution in two case studies: a monodimensional transport problem whose data and parameters correspond to a repository located at Mol (Belgium), chosen as the reference site for trial assessments of the feasibility of disposal of HLW in clay formations ${ }^{9}$; a case of bidimensional transport taken from the literature. ${ }^{10}$ The concentration output profiles obtained by the neural networks are compared to those given by a numerical code based on a finite element method (MCB2D, Multiple Cell Balance 2Dimensional). ${ }^{10}$

In Sections 2 and 3 the advection-dispersion model and the main features of the neural networks here employed are briefly described, for completion of the paper; in Section 4, the results of the networks' training for the two case studies considered are discussed, particularly with respect to accuracy and speed of the method. The paper closes with some concluding remarks in Section 5.

\section{The Advection-Dispersion Model}

The advection-dispersion model describes contaminant transport in groundwater by means of partial differential equations derived from energy and solute mass balances. The fundamental physical hypothesis of the model is that contaminant migration is due to two concomitant phenomena: advection and hydrodynamic dispersion. The process by which solutes are transported by the bulk motion of the flowing groundwater is known as advection. Owing to advection, nonreactive solutes are carried at an average rate equal to the average linear velocity of the fluid $v$. There is a tendency, however, for the solute to spread out from the path that it would be expected to follow according to the advective hydraulics of the flow system. This spreading phenomenon is called hydrodynamic dispersion. It causes dilution of the solute, and occurs because of two concurrent processes: mechanical dispersion and molecular diffusion, due respectively to disuniformities in the microscopic velocity field and in the solute concentration.

From the modeling point of view, a two-component fluid (solute and solvent) is here considered for simplicity. The solute mass balance in a representative elementary volume of the saturated porous medium leads to the following partial differential equation ${ }^{6}$ :

$$
\frac{\partial C}{\partial t}+\operatorname{div}\left(C v_{s}\right)=I
$$

where $C$ is the solute concentration $\left[\mathrm{kg} / \mathrm{m}^{3}\right], v_{S}$ its velocity and $I$ is the rate of production of the solute per unit volume $\left[\mathrm{kg} / \mathrm{m}^{3} \mathrm{~s}\right]$. Solute diffusion is described by the mass diffusion flux $\boldsymbol{J}$ :

$$
\boldsymbol{J}=C\left(\boldsymbol{v}_{s}-\mathbf{v}\right)
$$

where $v$ is the mean flow velocity and is related to the hydraulic gradient $\nabla h$ through Darcy's empirical law:

$$
\boldsymbol{v}=-\frac{\boldsymbol{K}}{n} \nabla h
$$

where $\boldsymbol{K}$ is the hydraulic conductivity of the medium and $n$ its porosity (given by the ratio between the void volume and the total volume); $\boldsymbol{v}$ is also called Darcy's velocity. Mass diffusion is assumed to occur according to Fick's law, which states the proportionality between the diffusion flux and the concentration gradient, through a proportionality constant called molecular diffusion coefficient $D_{d}$, so that diffusion occurs from higher to lower concentration zones ${ }^{6}$ :

$$
\boldsymbol{J}=-\rho D_{d} \nabla\left(\frac{C}{\rho}\right)
$$

where $\rho$ is the fluid density.

From Eqs. (2.2), (2.4) and the mass balance (2.1), the advection-dispersion equations are obtained. In Cartesian coordinates and assuming incompressibility of the fluid:

$$
\frac{\partial C}{\partial t}=\frac{\partial}{\partial x_{i}}\left(D_{i j} \frac{\partial C}{\partial x_{j}}\right)-\frac{\partial}{\partial x_{i}}\left(v_{i} C\right)+I .
$$

In Eq. (2.5), $D_{i j}$ is the element of a second-rank symmetric tensor $D$ called hydrodynamic dispersion coefficient, which is the sum of a mechanical dispersion term and of a molecular diffusion term (function of the 
molecular diffusion coefficient $D_{d}$ ). The hydrodynamic dispersion coefficient is also related to Darcy's velocity through a proportionality factor $\alpha$, called dispersivity.

The source/sink term $I$ in Eq. (2.5) can be due to various phenomena such as solute injections or extractions, chemical reactions and radioactive decay: here the attention is focused on the adsorption and ion exchange processes which occur at the interface between solid and liquid phases. The negative charges on a solid surface may attract the cations dissolved in the liquid phase, which may be adsorbed onto the solid surface thus reducing the contaminant concentration in the liquid; on the other hand, the contaminant ions in the solid may enter the liquid through the solid surface and increase the contaminant concentration in the liquid.

To account for these effects of interchange, we must simultaneously consider the mass balance within the solid and liquid phases:

$$
\begin{gathered}
\frac{\partial C}{\partial t}=\frac{\partial}{\partial x_{i}}\left(D_{i j} \frac{\partial C}{\partial x_{j}}\right)-\frac{\partial}{\partial x_{i}}\left(v_{i} C\right)+\frac{f(C, F)}{n} \\
\frac{\partial F}{\partial t}=-\frac{f(C, F)}{1-n}
\end{gathered}
$$

where $F\left[\mathrm{~kg} / \mathrm{m}^{3}\right]$ is the contaminant concentration in the solid and $f(C, F)\left[\mathrm{kg} / \mathrm{m}^{3} \mathrm{~s}\right]$ is the mass of contaminant transferred from solid to liquid, per unit time and per unit volume of porous media.

Several expressions have been suggested for $f(C, F)$ for different adsorption cases; in this paper, the usual linear equilibrium isotherm relationship is considered which assumes that the solute concentration in the solid phase is directly proportional to the concentration in the liquid phase: $F=\beta C$, where $\beta$ is known as the distribution coefficient. With this hypothesis, the contaminant concentration in the liquid phase turns out to be described by the following equation:

$$
\frac{\partial C}{\partial t}=\frac{\partial}{\partial x_{i}}\left(\frac{D_{i j}}{R} \frac{\partial C}{\partial x_{j}}\right)-\frac{\partial}{\partial x_{i}}\left(\frac{v_{i}}{R} C\right)
$$

where $R=1+\frac{1-n}{n} \beta$ is called the retardation factor

and describes the slowing down in contaminant propagation caused by adsorption. Since $R>1$, both the hydrodynamic dispersion coefficient and the mean flow velocity are decreased and, hence, the dispersion process is weakened.

\section{The Supervised, Feedforward Neural Network}

Neural networks are systems composed of simple processing elements (nodes) linked by weighted connections. In its simplest and most popular form, the multilayered feedforward neural network consists of three layers of processing elements: the input, the hidden and the output layers, with $n_{i}, n_{h}$ and $n_{o}$ nodes respectively. The signal, which consists of $n_{i}$ real values feeding the input nodes, is processed forwards from the input to the output layer (Fig. 3.1). Each node collects the sum of the output values, weighted by the connection strengths, from all the nodes of the preceding layer, processes this information through a sigmoidal activation function $f(x)=\left(1+e^{-x}\right)^{-1}$ and then delivers the result to all connections towards the nodes of the successive layer. In the present work, both input and hidden layers have the additional bias node which acts as a threshold in the argument of the activation function and whose output always equals unity. The natural choice of the number of nodes $n_{o}$ in the output layer is to take as many nodes as the number of quantities to be estimated in the problem. On the contrary, there are no set rules for choosing the numbers of nodes in the other two layers: here we followed a rational criterion for which the number of input nodes $n_{i}$ has been chosen equal to that of the data variables which affect the output, while the number of hidden nodes $n_{h}$ has been kept as low as possible in view of the need of building fast-computing models.

The values of the connection weights are randomly initialized and then fine-tuned through an iterative training procedure. In this work, the popular error backpropagation algorithm has been adopted for the training. ${ }^{11}$ In short, a set of $N_{p}$ input and corresponding output patterns are repeatedly presented to the network and the values of the connection weights are iteratively adjusted so as to minimise the average squared output deviation error function, or energy function, over all training patterns and all $n_{o}$ outputs, defined as:

$$
\mathcal{E}=\frac{1}{2 N_{p} n_{o}} \sum_{p=1}^{N_{p}} \sum_{l=1}^{n_{o}}\left(t_{p l}-o_{p l}\right)^{2}
$$

where $t_{p l}$ and $o_{p l}$ are the true and the network-computed values of the $l$-th output node, to the $p$-th pattern presented.

Through this training procedure, the network is able to build an internal representation of the input/output mapping of the problem under investigation. 
The success of training strongly depends on the normalization of the data and on the choice of the training parameters. Concerning the normalization, the $N_{p}$ patterns of the training set can be thought of as the rows of an $\left(N_{p}, n_{i}\right)$ matrix; since the $n_{i}$ data in each pattern may be largely different, it is common to suitably normalize the data appearing in each column, by linearly mapping them in a given interval (e.g. [0.2, $0.8]$ ).

After the training is completed, when new input patterns are presented the network recalls the information stored in the connection weights to produce the corresponding output, coherent with the internal representation of the input/output mapping.

Notice that the nonlinearity of the sigmoidal function of the processing elements allows the neural networks to learn arbitrary nonlinear mappings. Moreover, each node acts independently of all the others and its functioning relies only on the local information provided through the adjoining connections. In other words, the functioning of one node does not depend on the states of those other nodes to which it is not connected. This allows for efficient distributed representation and parallel processing, and for an intrinsic fault-tolerance and generalization capability. These attributes render the neural networks a powerful tool for signal processing and nonlinear mappings. ${ }^{12-14}$

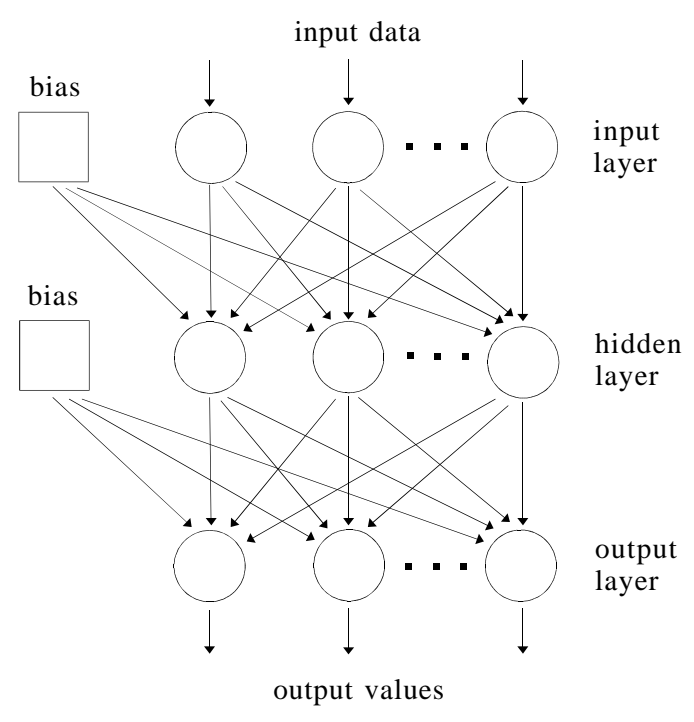

Fig. 3.1. Scheme of a three-layered, feedforward neural network.

\section{Simulating Contaminant Transport via Neural Networks}

In this Section, neural networks are trained to simulate the transport of contaminant in groundwater for two case studies of monodimensional and bidimensional aquifers. Through an appropriate training procedure the networks learn to predict the values of contaminant concentration (output) at given locations and times, from knowledge of the input hydro-geological parameters of the medium (input).

\subsection{Monodimensional case}

A transport problem in a monodimensional, homogeneous, isotropic and semi-infinite aquifer is considered; the hydro-geological characteristic parameters are relative to the upper aquifer of the Mol site in Belgium. ${ }^{9}$ The study is focused on the transport of the radionuclide $\mathrm{Np}-237$, selected among others because of its nuclear, physical and transport

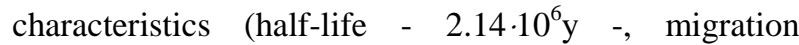
capability, radiotoxicity) which make it a most hazardous contaminant for radiological impacts.

The source term is given by a point source of $1.5 \cdot 10^{11} \mathrm{~Bq} / \mathrm{m}^{3}$ of $\mathrm{Np}-237$ located in the origin of the aquifer: this quantity corresponds to the content of 1 ton reprocessed fuel which dissolves completely and instantaneously after the canister corrosion.

The objective is to train a set of neural networks for the prediction of the Np-237 contaminant spatial distribution at two times of interest for short and long term safeguard respectively: $\mathrm{t}=50 \mathrm{~d}$ and $\mathrm{t}=10^{4} \mathrm{y}$.

Due to the heterogeneities in the porous medium, the hydro-geological parameters (hydraulic conductivity $K$, Darcy's velocity $v$, retardation factor $R$, dispersivity $\alpha)$ are affected by uncertainty: this is taken into account by assigning proper probability distributions to the various parameters. ${ }^{9}$

To analyze the uncertainty in the output concentration due to the uncertainty in the input parameters, a sensitivity study has been performed with the objective of establishing an upper bound of acceptable network approximation error, defined as the discrepancy between the network predicted output and the true concentration value divided by the true value (relative error). A high-order Monte Carlo sensitivity analysis ${ }^{15}$ was performed varying one parameter at the time within its range of variability and keeping all other parameters at three different levels corresponding to 
their central, minimum and maximum values. A large number of preliminary tests allowed us to identify Darcy's velocity $v$ and the retardation factor $R$ as the major contributors to the output concentration spatial distribution variability, defined in terms of the sensitivity index $I_{S}$ :

$$
I_{s}=\frac{1}{N} \sum_{i=1}^{N} \frac{\left|C_{\max }\left(x_{i}\right)-C_{\text {min }}\left(x_{i}\right)\right|}{C_{\text {med }}\left(x_{i}\right)}
$$

where $x_{i}, i=1,2, \ldots, N$, is the coordinate of concentration observation and $C_{\text {min }}, C_{\text {med }}$ and $C_{\max }$ are the concentrations corresponding to the minimum, medium and maximum parameters' values, respectively. A more detailed analysis showed that the variability of $v$ leads to an output variability of up to $200 \%$, whereas $R$ causes an uncertainty in the output of about $85 \%$. This latter value was taken as the upper limit to the acceptable relative error in the neural networks predictions.

For what concerns the prediction at $\mathrm{t}=50 \mathrm{~d}$, the patterns for training have been generated by means of $N s=200$ simulations with the code MCB2D which resolves numerically the advection-dispersion equations for a given set of input data. Each pattern contains five inputs, namely the coefficient of adsorption $\beta$, the hydraulic conductivity $K$, the longitudinal dispersivity $\alpha_{L}$, the effective velocity $v / R$ and the coordinate of the concentration observation point $x_{p}$, and one output, the concentration value at the observation point, $C\left(x_{p}\right)$. Each one of the 200 simulations generates $N_{p}$ patterns, one for each observation point $x_{p}, p=1,2, \ldots, N_{p}$.

As a first attempt, one single neural network was trained for the whole range of variability of the input parameters, but this led to poor specialization of the network and corresponding relative prediction errors of the order of $100 \%$. To specialize the network models, training was repeated on narrower subranges of the input parameter $v / R$, which in the previous sensitivity analysis was found to be the most critical for the output variability. More precisely, four networks were devised and their trainings optimized with respect to the network parameters, learning rate $\eta$, momentum $\alpha$, number of hidden nodes $n_{h}$, pattern repetitions $n_{r}$. The results of the trainings are shown in Table 4.1 , in which $\varepsilon$ indicates the network average relative error on the patterns of the training set, defined as:

$$
\varepsilon=\frac{1}{N_{s} N_{p}} \sum_{s=1}^{N_{s}} \sum_{p=1}^{N_{p}} \frac{C_{\text {true }}\left(x_{p}^{s}\right)-C_{\text {network }}\left(x_{p}^{s}\right)}{C_{\text {true }}\left(x_{p}^{s}\right)}
$$

where $x_{p}^{s}$ is the $p$-th concentration observation point in the $s$-th simulation.

After training, the networks have been tested on 6300 new patterns; Table 4.1 shows the parameter values optimizing the networks and the average relative prediction error for each network; Figures 4.1-4.4 show the good match between the numerical and the networks solutions for parameter values within the four different ranges.

\begin{tabular}{|c|c|c|c|c|c|c|}
\hline $\begin{array}{c}\text { Network } \\
\text { (v/R range } \\
[\mathrm{m} / \mathrm{d}])\end{array}$ & $\eta$ & $\alpha$ & $\mathbf{n}_{\mathrm{h}}$ & $\mathbf{n}_{\mathbf{r}}$ & $\varepsilon$ & $\varepsilon_{\text {test }}$ \\
\hline $\begin{array}{c}1 \\
\left(4 \cdot 10^{-5} \div 6 \cdot 10^{-4}\right)\end{array}$ & 0.4 & 0.8 & 7 & 10000 & $15.79 \%$ & $15.51 \%$ \\
\hline $\begin{array}{c}\mathbf{2} \\
\left(5 \cdot 10^{-4} \div 1.2 \cdot 10^{-3}\right)\end{array}$ & 0.6 & 0.9 & 6 & 50000 & $12.20 \%$ & $12.19 \%$ \\
\hline $\begin{array}{c}\mathbf{3} \\
\left(1 \cdot 10^{-3} \div 2 \cdot 10^{-3}\right)\end{array}$ & 0.6 & 0.9 & 6 & 50000 & $10.34 \%$ & $10.93 \%$ \\
\hline $\begin{array}{c}\mathbf{4} \\
\left(1.5 \cdot 10^{-3} \div 3 \cdot 10^{-3}\right)\end{array}$ & 0.6 & 0.9 & 8 & 50000 & $5.57 \%$ & $6.26 \%$ \\
\hline
\end{tabular}

Table 4.1. Networks parameters and performances.

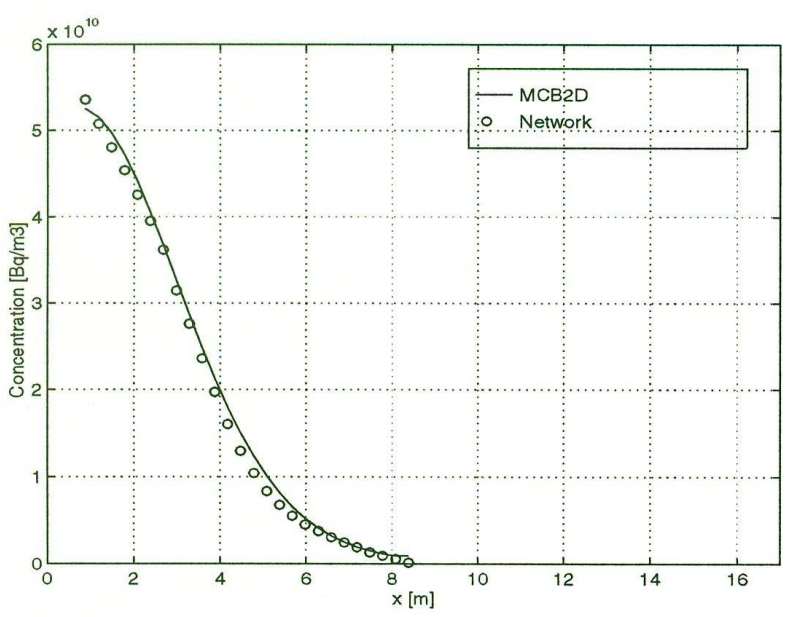

Fig. 4.1. Network $1, v / R=4.34 \cdot 10^{-4} \mathrm{~m} / \mathrm{d}, \varepsilon_{\text {test }}=11.9 \%$ 


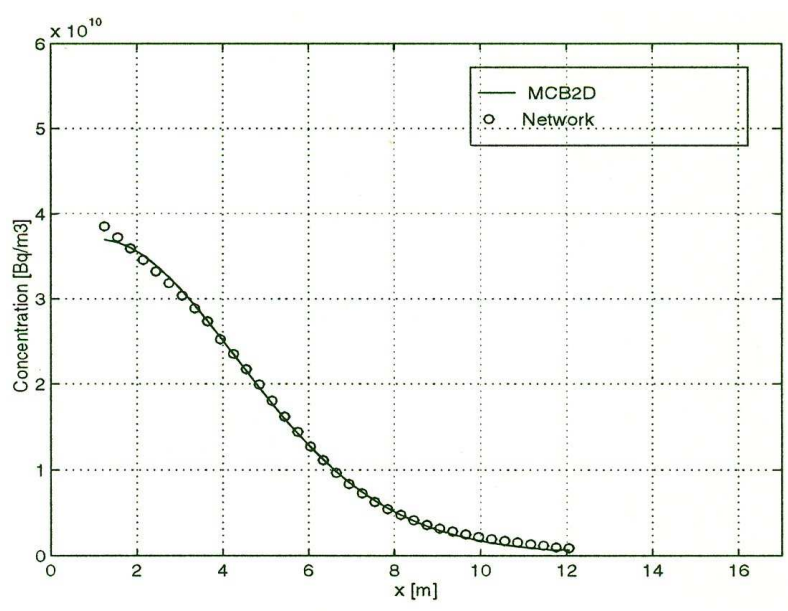

Fig. 4.2. Network $2, \mathrm{v} / \mathrm{R}=6.45 \cdot 10^{-4} \mathrm{~m} / \mathrm{d}$, ctest $=10.7 \%$

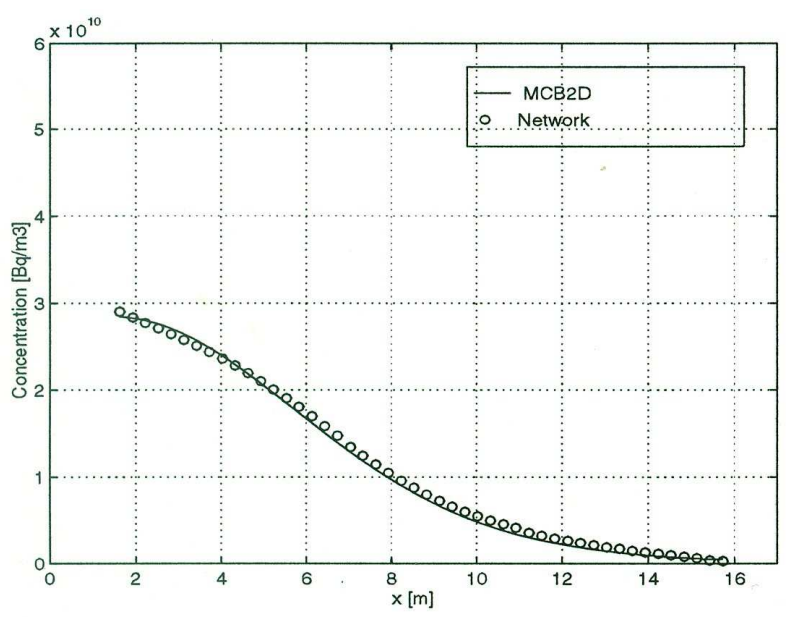

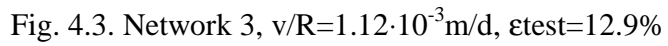

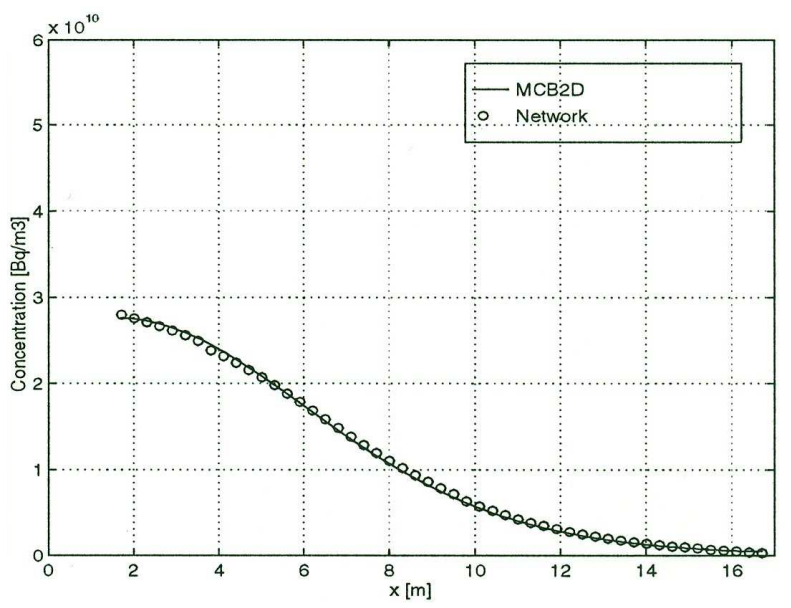

Fig. 4.4. Network $4, \mathrm{v} / \mathrm{R}=2.58 \cdot 10^{-3} \mathrm{~m} / \mathrm{d}$, ctest $=5.0 \%$

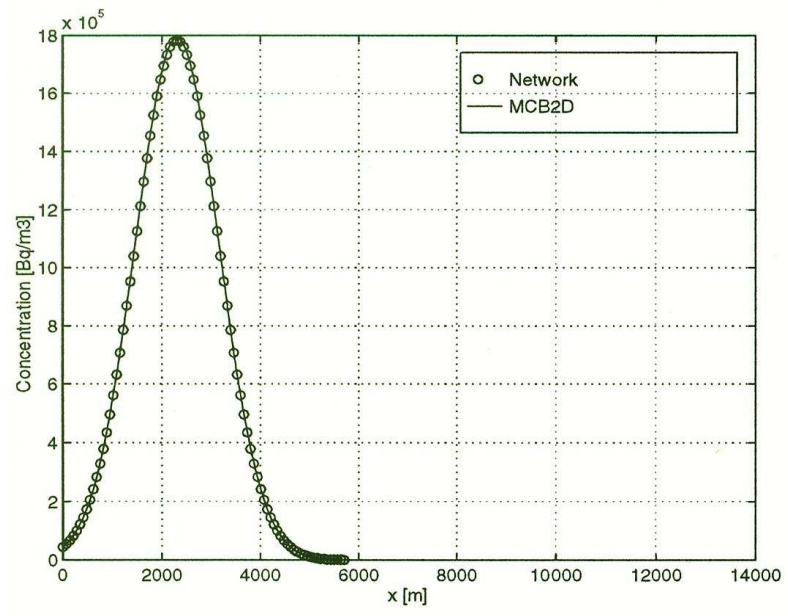

Fig. 4.5. Network $1, \mathrm{v} / \mathrm{R}=5.6 \cdot 10^{-4} \mathrm{~m} / \mathrm{d}$, ctest $=0.4 \%$

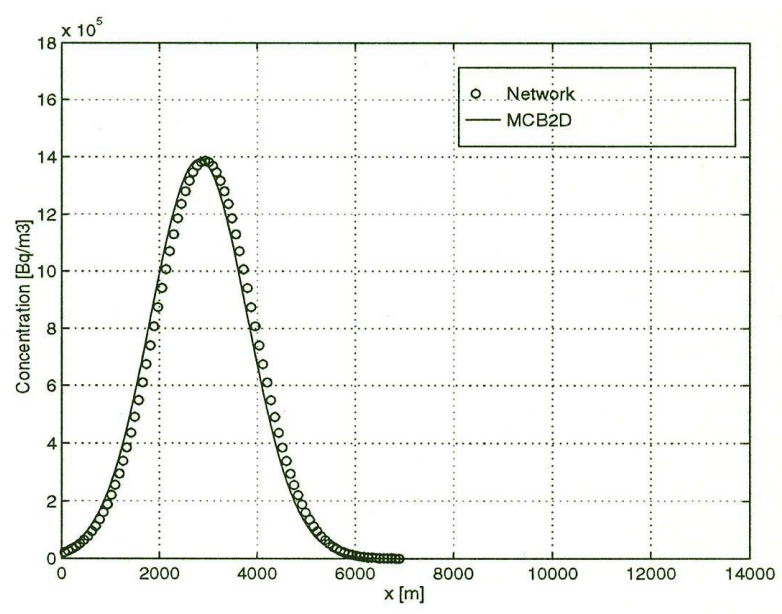

Fig. 4.6. Network 2, v/R=0.8 $10^{-3} \mathrm{~m} / \mathrm{d}$, عtest $=0.36 \%$

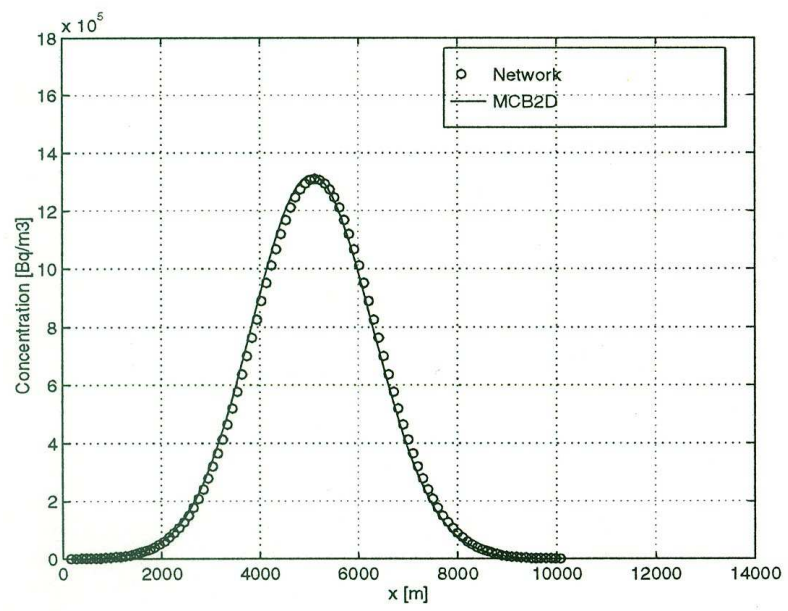

Fig. 4.7. Network $3, \mathrm{v} / \mathrm{R}=1.3 \cdot 10^{-3} \mathrm{~m} / \mathrm{d}$, ctest $=0.89 \%$ 


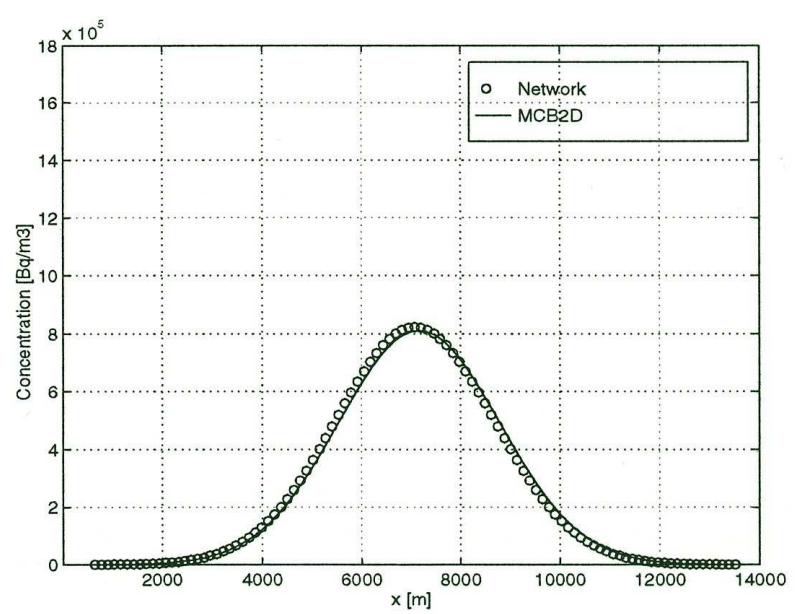

Fig. 4.8. Network $4, \mathrm{v} / \mathrm{R}=1.9 \cdot 10^{-3} \mathrm{~m} / \mathrm{d}$, ctest $=1.51 \%$

For the case of $t=10^{4} \mathrm{y}$, a different strategy was followed for the training of the neural networks: based on the gaussian shape of the concentration profiles, the networks have been trained to give in output the parameters $\mu, \sigma$ and the area $A$ of the gaussian profile, the inputs being the coefficient of adsorption $\beta$, the hydraulic conductivity $K$, the longitudinal dispersivity $\alpha_{L}$ and the effective velocity $v / R$.

A set of $N_{S}=200$ simulations were performed with the MCB2D code to generate the training patterns. The previously successful strategy of training four specialized networks on the subranges of $v / R$ was again followed and led to satisfactory results on the training set, with prediction errors ranging from $0.5 \%$ to $1.5 \%$, for networks parameters $\eta=0.6, \alpha=0.8, n_{h}=5, n_{r}=50000$.

Tests with about 200 new patterns produced similar errors. Figures 4.5-4.8 show the good match between the numerical and the network curves, for the four specialized networks.

\subsection{Bidimensional case}

An irregular bidimensional geometry simulating a confined aquifer between two rivers has been considered (Figure 4.9). ${ }^{10}$ The flow is assumed to be bidimensional, with the upstream river being an instantaneous source of contamination injecting into the aquifer a concentration of $2000 \mathrm{~Bq} / \mathrm{m}^{3}$.

The prediction of contamination at every location in the aquifer is used to study altered evolution scenarios in which the hydro-geological parameters values are modified. To this aim, two main steps have been undertaken. First, the evolution of the contaminant

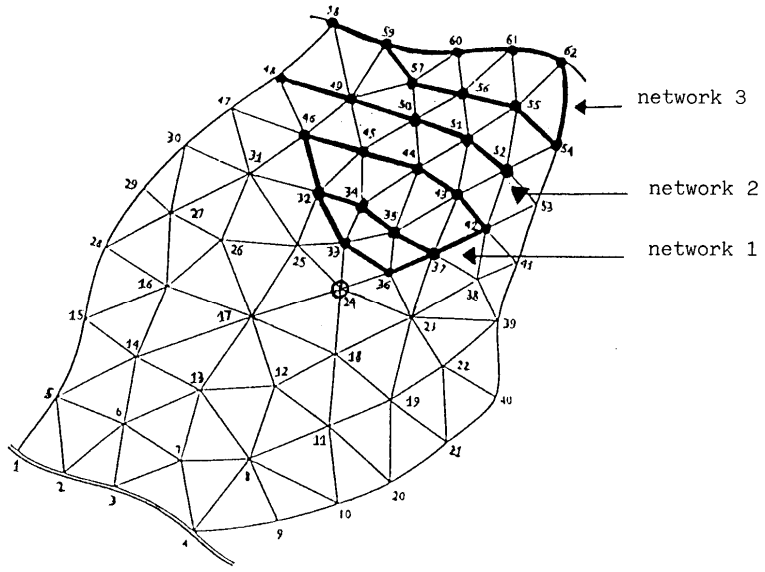

Fig. 4.9.Aquifer configuration and finite element discretization of the problem.

concentration must be followed up to the time of occurrence of the altering event, under the normal scenario conditions but still accounting for the uncertainty in the hydro-geological parameters. At the time of the event occurrence, the concentration values at each point in the aquifer constitute the initial conditions for the successive evolution of the contaminant in the altered scenario, which is characterized by different values of the hydro-geological parameters with different uncertainty distributions. The second step then consists in determining the contribution of each contamination sources at every point in the aquifer to the evolution of the contamination profile in a given control point, for parameter values sampled from the distributions characterizing the site after the altering event.

In the application considered, the stochastic event has been assumed to occur at $t=3000 \mathrm{y}$ and the control point has been taken in correspondence of node 24 in Figure 4.9.

For the prediction of the contaminant spatial distribution at $\mathrm{t}=3000 \mathrm{y}$, a set of patterns were generated through 300 simulations with the MCB2D code; each pattern contains five inputs, coefficient of adsorption $\beta$, hydraulic conductivity $K$, longitudinal dispersivity $\alpha_{L}$, effective velocity $v / R$ and the coordinate of the observation node $x_{n}$, and one output, the concentration value in the observation node $C\left(x_{n}\right)$. Each simulation gives $N$ patterns, one for each observation node $x_{n}, n=1$, $2, \ldots, N$.

To predict the concentration profile evolution in the control node 24 , the networks were trained to predict the 
concentration time evolutions in every point of the aquifer contributing to the contamination in 24 (i.e. all the nodes in the upper part of the aquifer, given that the flux is directed downward). Because of the great variability (two orders of magnitude) of the concentration values on the different nodes in the domain of interest, this was divided in three layers, containing nodes $32-37$ and 42-46, nodes $48-53$, nodes 54-62, respectively (see Figure 4.9); one neural network was then trained specifically on each of them.

The parameters for the optimal networks training and the average relative errors between the numerical and the networks outputs, are summarized in Table 4.2. Testing was performed on 3900 new patterns; Table 4.2 shows the parameter values optimizing the networks and the average relative prediction error for each network; Figures 4.10-4.12 show the good match between the true (numerical code) and the predicted NN profiles, for three of the test patterns.

Table 4.2. Networks parameters and performances.

\begin{tabular}{|c|c|c|c|c|c|c|}
\hline & $\boldsymbol{\eta}$ & $\boldsymbol{\alpha}$ & $\mathbf{n}_{\mathbf{h}}$ & $\mathbf{n}_{\mathbf{r}}$ & $\boldsymbol{\varepsilon}$ & $\boldsymbol{\varepsilon}_{\text {test }}$ \\
\hline $\begin{array}{c}\text { Network } \\
\mathbf{1}\end{array}$ & 0.6 & 0.8 & 8 & 50000 & $18.76 \%$ & $18.35 \%$ \\
\hline $\begin{array}{c}\text { Network } \\
\mathbf{2}\end{array}$ & 0.6 & 0.8 & 5 & 30000 & $18.55 \%$ & $19.80 \%$ \\
\hline $\begin{array}{c}\text { Network } \\
\mathbf{3}\end{array}$ & 0.6 & 0.7 & 6 & 60000 & $33.25 \%$ & $31.84 \%$ \\
\hline
\end{tabular}

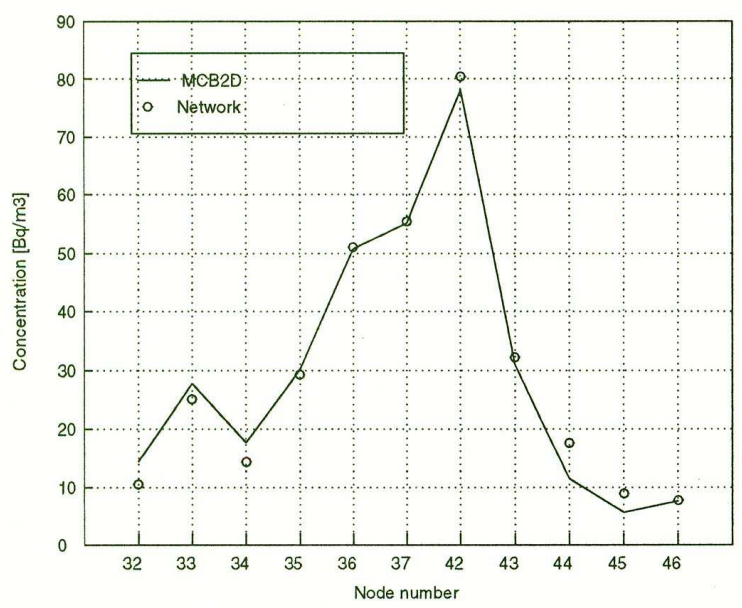

Fig. 4.10. Network 1, trained on nodes 32-37 and 42-46 $v / R=8.8 \cdot 10^{-4} \mathrm{~m} / \mathrm{d}, \varepsilon_{\text {test }}=16.2 \%$

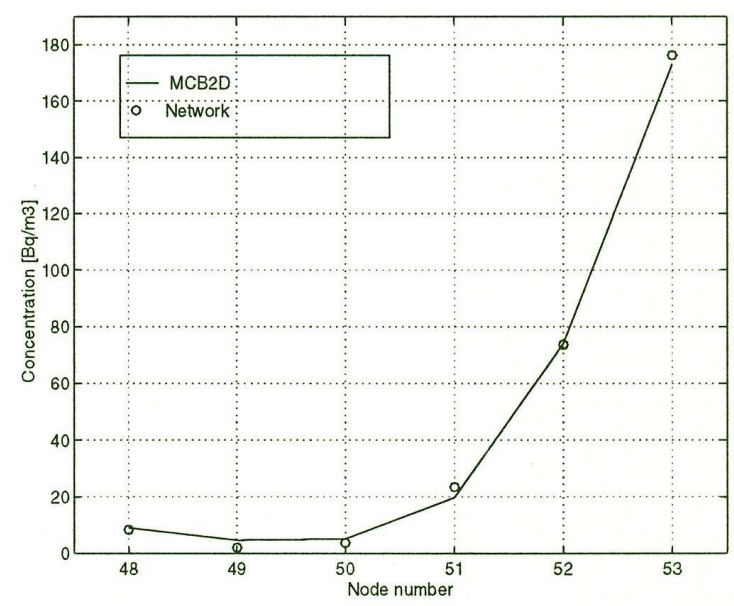

Fig. 4.11. Network 2, trained on nodes 48-53, $v / R=7.4 \cdot 10^{-4} \mathrm{~m} / \mathrm{d}, \varepsilon_{\mathrm{test}}=18.9 \%$

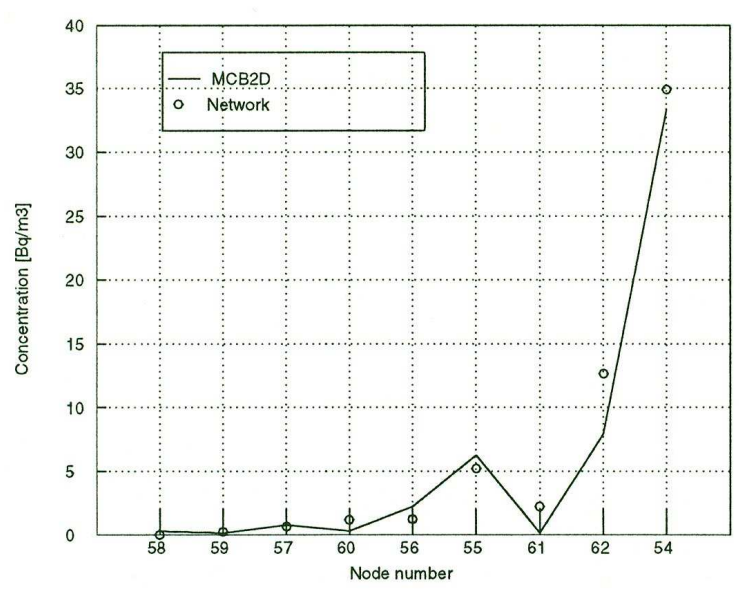

Fig. 4.12. Network 3, trained on nodes 54-62, $v / R=1.1 \cdot 10^{-3} \mathrm{~m} / \mathrm{d}, \varepsilon_{\text {test }}=31.1 \%$

The successive step of the analysis regarded the study of the time evolution of contamination in node 24, due to the neighbouring nodes. For simplicity, the contribution of only 3 nodes, namely 45,52 and 56 , as point sources of $2000 \mathrm{~Bq} / \mathrm{m}^{3}$, was considered; one network was trained to predict the resulting concentration in node 24 due to the initial contamination in each of the three nodes 45,52 and 56, respectively, on a set of 24 time instants ranging from 100 to 10000 years.

Training patterns were generated through 300 simulations with the MCB2D code, each pattern containing the five following inputs: coefficient of adsorption $\beta$, hydraulic conductivity $K$, longitudinal dispersivity $\alpha_{L}$, effective velocity $v / R$ and the 
observation time instant $t_{p}$, and one output, the concentration value in node 24 at the observation time $t_{p}, C\left(t_{p}\right)$. Each simulation gives $N_{t}=24$ patterns, one for each observation time $t_{p}, p=1,2, \ldots, N_{t}$.

Table 4.3 shows the parameter values optimizing the networks behaviour and the average relative prediction error for each network.

Table 4.3: Networks parameters and performances.

\begin{tabular}{|c|c|c|c|c|c|c|}
\hline & $\boldsymbol{\mu}$ & $\boldsymbol{\alpha}$ & $\mathbf{n}_{\mathbf{h}}$ & $\mathbf{n}_{\mathbf{r}}$ & $\boldsymbol{\varepsilon}$ & $\boldsymbol{\varepsilon}_{\text {test }}$ \\
\hline $\begin{array}{c}\text { Network } \\
\mathbf{N} 45\end{array}$ & 0.5 & 0.8 & 5 & 30000 & $15.36 \%$ & $19.08 \%$ \\
\hline $\begin{array}{c}\text { Network } \\
\mathbf{N} 52\end{array}$ & 0.6 & 0.8 & 5 & 50000 & $6.11 \%$ & $5.32 \%$ \\
\hline $\begin{array}{c}\text { Network } \\
\mathbf{N} 56\end{array}$ & 0.6 & 0.8 & 4 & 30000 & $13 \%$ & $15.98 \%$ \\
\hline
\end{tabular}

A test on 3600 new patterns gave similar results, as shown in Table 4.3. Figures 4.13-4.15 show the satisfactory approximation obtained with the three networks.

\section{Conclusions}

In this paper, the possibility of using neural networks for predicting the space-time distribution of contaminant in groundwater has been demonstrated. The approach undertaken to guarantee the satisfactory accuracy has consisted in training a set of different networks to predict the contaminant concentration at given times and locations, starting from knowledge of the hydro-geological parameters of the hosting medium.

The application to a monodimensional and a bidimensional case has led to predictive relative errors of about $10-20 \%$, which are considered acceptable in consideration of the uncertainties which propagate from the model parameters to its outputs.

Furthermore, once trained the neural networks take a negligible time to compute the output, contrary to the numerical codes; as accounting for uncertainties in probabilistic performance assessments of radioactive waste deposits requires a large number of simulations to cover the variability ranges of the input parameters, these savings in computer time can be of fundamental importance.

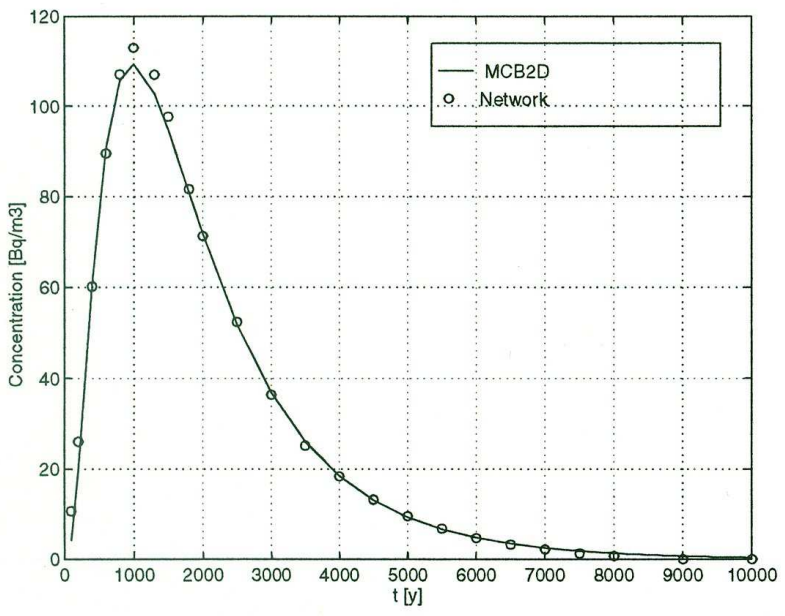

Fig. 4.13: Network with source on node $45, v / R=9.4 \cdot 10^{-4} \mathrm{~m} / \mathrm{d}$, $\varepsilon_{\text {test }}=14.1 \%$

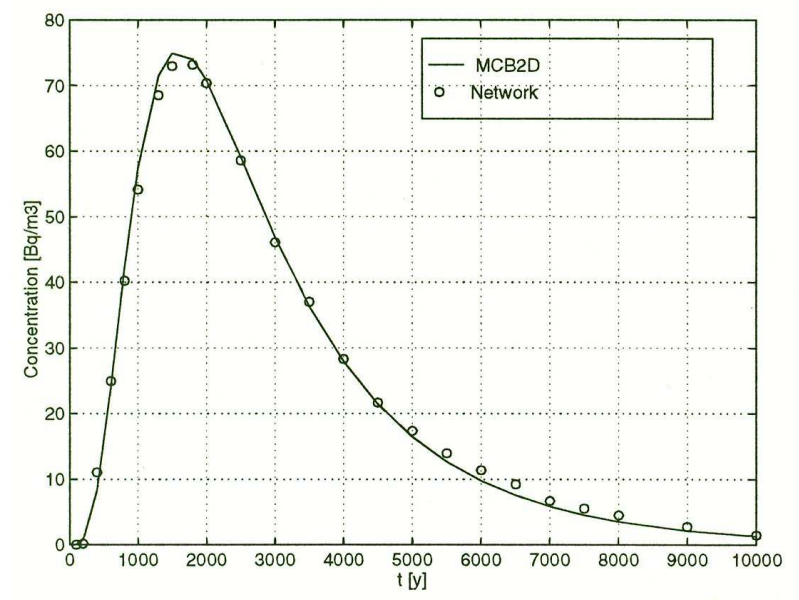

Fig. 4.14. Network with source on node $52, v / R=8.5 \cdot 10^{-4} \mathrm{~m} / \mathrm{d}$, $\varepsilon_{\text {test }}=4.7 \%$

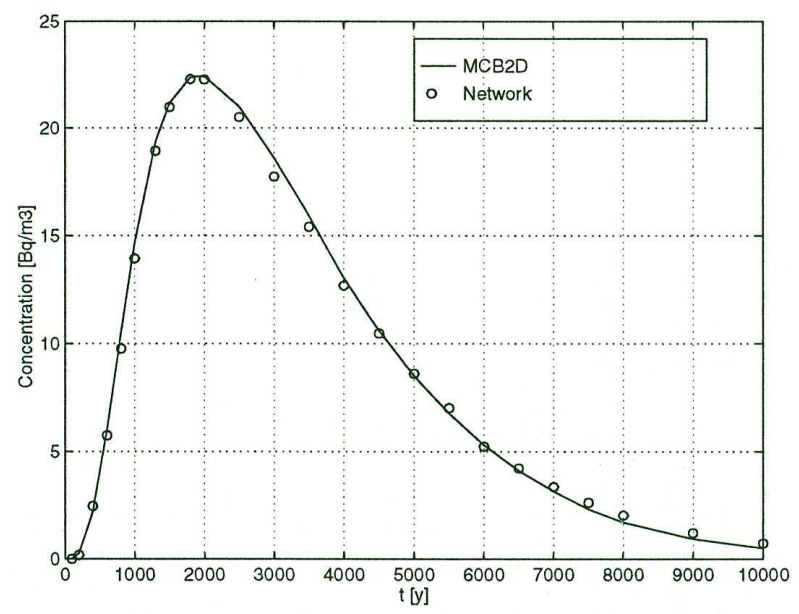

Fig. 4.15. Network with source on node $56, v / R=8.5 \cdot 10^{-4} \mathrm{~m} / \mathrm{d}$, $\varepsilon_{\text {test }}=10.2 \%$ 


\section{Acknowledgements}

The author wishes to thank for their contribution to the work Professor M. Marseguerra and Ingg. R. De Paoli and C. Soffientini, formerly with the Department of Energy of the Politecnico di Milano.

\section{References}

1. IAEA, Near surface disposal of radioactive waste, IAEA Safety Standards Series-Requirements, WS-R-1 (Vienna 1999).

2. IAEA, Safety assessment of near surface disposal of radioactive waste, IAEA Safety Standards Series - Safety Guide, WS-G-1.1 (Vienna 1999).

3. NUREG-1573, A performance assessment methodology for low-level radioactive waste disposal facility, (October 2000).

4. M.-S. Yim and S. A. Simonson, Performance Assessment Models for Low Level Radioactive Waste Disposal Facilities: A Review, Progress in Nuclear Energy, Vol. 36, No 1, (2000) 1-38.

5. Jon C. Helton and Cedric J. Sallaberry, Conceptual basis for the definition and calculation of expected dose in performance assessment for the proposed high-level radioactive waste repository at Yucca Mountain, Nevada, Reliability Engineering and System Safety doi:10.1016/j.ress.2008.06.011, Vol. 94, N. 3, (2008) 677-698.

6. R. A. Freeze and J. A. Cherry, Groundwater, (PrenticeHall Inc., Englewood Cliffs, New Jersey, 1979).

7. R. Willis and W. W. G. Yeh, Groundwater System Planning and Management, (Prentice.Hall Inc., Englewood Cliffs, New Jersey, (1987).

8. Jon C. Helton and Cedric J. Sallaberry, Computational implementation of sampling-based approaches to the calculation of expected dose in performance assessments for the proposed high-level radioactive waste repository at Yucca Mountain, Nevada, Reliability Engineering and System Safety, doi:10.1016/j.ress.2008.06.018, Vol. 94, N. 3, (2008) 699-722.

9. PAGIS, Performance Assessment of Geological Disposal System for HLW (1988). In Clay Formation, EUR 11776 (EN). Summary, EUR 11775 (EN).

10. N. Z. Sun, Mathematical Modelling of Groundwater Pollution, (Springer-Verlag, New York, 1996).

11. D. E Rumelhart. and J. L. McClelland, Parallel Distributed Processing, Vol. 1, (MIT Press, Cambridge, 1986).

12. P. Vroman, L. Koehl, X. Zeng and T. Chen, Designing Structural Parameters of Nonwovens Using Fuzzy Logic and Neural Networks, International Journal of Computational Intelligence Systems, 1(4), (2008) 340352.

13. N. Castin, L. Malerba and R. Pinheiro Domingos, Use of Computational Intelligence for the Prediction of Vacancy Migration in Atomistic Kinetic Monte Carlo
Simulations", International Journal of Computational Intelligence Systems, 1(4), (2008) 340-352.

14. S. Gowrishankar and P.S. Satyanarayana, Neural Network Based Traffic Prediction for Wireless Data Networks, International Journal of Computational Intelligence Systems, 1(4), (2008) 379-389.

15. J. C. Helton, Uncertainty and Sensitivity Analysis Techniques for Use in Performance Assessment for Radioactive Waste Disposal, Reliability Engineering and System Safety, 42 (2-3), (1993) 327-367. 